\title{
Training method for animal-based measures in dairy cattle welfare assessments
}

\author{
S. L. Croyle, C. G. R. Nash, C. Bauman, S. J. LeBlanc, D. B. Haley, D. K. Khosa, and D. F. Kelton ${ }^{1}$ \\ ${ }^{*}$ Department of Population Medicine, University of Guelph, Ontario, N1G 2W1 Canada
}

\begin{abstract}
Quantitative assessments of animal welfare are increasingly being used in the dairy industry. It is important to have good precision and accuracy within and among assessors. This study explored the effectiveness of a 3-d training workshop for animal-based measures $(\mathrm{ABM})$ of welfare in dairy cows, in which 14 people were trained to evaluate $6 \mathrm{ABM}$, specifically hock injuries $(\mathrm{HI})$, lameness (LM), body condition score (BCS), and udder, flank, and leg cleanliness (collectively CLN). All scoring systems were modified to a dichotomous outcome, acceptable or unacceptable. Acceptable HI included no swelling or hair loss, unacceptable HI included swelling or scab; acceptable BCS was $>2$ on a 5-point scale, unacceptable BCS was $\leq 2$. Acceptable CLN was up to minor splashing, unacceptable CLN was distinct plaques to a solid manure plaque. Lameness was evaluated using locomotion score or in-stall lameness score (SLS) in tiestalls; unacceptable LM was $\geq 3$ (lameness score) on a 5 -point scale, where 3 equals mild lameness or $\geq 2$ of 4 behavioral in-stall lameness score indicators were detected. Classroom instruction took place on d 1 of training. Day 2 consisted of group assessment of LM ( $\mathrm{n}=25$ cows), and HI, CLN, and BCS ( $\mathrm{n}=30$ cows), and individual assessment of HI, CLN, and BCS ( $\mathrm{n}=20$ cows) were performed. Day 3 included individual assessments of HI, CLN, and BCS ( $\mathrm{n}=33$ cows $)$, and individual video assessment of LM $(\mathrm{n}=27$ cows $)$. An additional training video for LM was sent to trainees 3 wk after the workshop, and another follow-up assessment of LM took place via video $(\mathrm{n}=$ 37 cows). Repeatability and accuracy of the trainees was assessed using Fleiss's $\kappa(\mathrm{FK})$ and Byrt's $\kappa(\mathrm{BK})$ to examine group-level inter-rater agreement and experttrainee agreement, respectively. The kappa systems use a scale of poor $(<0)$, slight $(0.01$ to 0.20$)$, fair $(0.21$ to 0.40$)$, moderate (0.41 to 0.61 ), substantial (0.61 to
\end{abstract}

Received January 18, 2018.

Accepted June 19, 2018.

${ }^{1}$ Corresponding author: dkelton@uoguelph.ca
$0.80)$, or almost perfect (0.81 to 1.00). At the conclusion of the workshop, FK was 0.66 for HI and 0.43 for LM, and BK mean (and range) was 0.85 (0.63 to 1.00) for HI and 0.66 (0.56 to 0.85) for LM. Each trainee achieved perfect agreement for BCS $[\mathrm{BK}$ mean $=1.00$ (1.00 to 1.00)] and each trainee achieved almost perfect agreement for CLN [BK mean 0.90 (0.82 to 0.94)]. After the follow-up video and 3 wk of experience, trainees achieved a FK of 0.66 and a BK mean of 0.74 (0.62 to 0.89 ) for LM. In conclusion, multiple assessors can achieve substantial agreement for ABM with adequate training.

Key words: lameness, hock injuries, inter-rater reliability

\section{INTRODUCTION}

There is increased interest in scoring groups of cows using animal-based measures (ABM) such as hock injury, lameness, cleanliness, and body condition to assess welfare on dairy farms for the purposes of research, consulting, animal welfare auditing, and benchmarking (Whay et al., 2003; Rushen et al., 2011; Vasseur et al., 2013). Previous studies have explored training for various individual $\mathrm{ABM}$, including hock injury scoring (Gibbons et al., 2012), gait scoring or lameness (Thomsen et al., 2008; Gibbons et al., 2014), and BCS (Vasseur et al., 2013). To date, no studies have explored training protocols for cleanliness of the leg, flank, and udder.

Assessor training is a useful tool to increase the precision and accuracy, thereby providing more consistent and valid results across farms. This allows the assessment to yield the same results with different assessors, and that these results reflect the assessment of an expert in the field (Lievens, 2001). It has been suggested that methods of scoring can be taught in a group format for injury scoring in dairy cattle, and that these individuals can achieve high levels of repeatability between raters (Gibbons et al., 2014). To our knowledge, no studies have described the process of training numerous people for multiple ABM at one time, which would be more reflective of the way assessments and training are 
likely to be conducted. Additionally, no studies have described, in detail, the amount of time spent teaching and practicing during the training process, which would be valuable information to replicate training programs and achieve similar results. To more rigorously assess interobserver reliability and accuracy of the scoring, it is recommended to calculate Cohen's $\kappa$ between 2 raters (i.e., rater vs. expert), Fleiss's $\kappa$ when there are more than 3 raters, and Byrt's $\kappa$ when the prevalence of outcomes is low, as well as reporting the raw percent agreement for comparison. A new program in Canada requires that all Canadian dairy farmers have an assessment done for key ABM. Veterinarians are expected to help farmers in their role as advisors; however, no current training format exists to prepare advisors for all the required ABM.

Our objective was to explore the effectiveness of a $3-d$ training workshop for ABM of welfare in dairy cows, in which 14 people could be trained to evaluate 6 ABM: hock injuries (HI), lameness (LM), BCS, and udder, flank, and leg cleanliness (CLN). The aim of the study was to provide a training format for future workshops to promote high accuracy and inter-rater agreement for multiple measures of animal welfare.

\section{METHODS}

\section{Scoring Constructs}

Multiple scoring systems are available for each ABM; however, the methodology for the current study was derived from Dairy Farmers of Canada's proAction Animal Care Assessment because this is the uniform scoring system implemented in a mandatory, industry-wide Canadian program (Dairy Farmers of Canada, 2015). All detailed scoring protocols discussed below were taken directly from Canadian Dairy Research Portal's Animal Comfort Tool, section "How to score injury, cleanliness, body condition, and lameness" (Canadian Dairy Research Portal, 2018). The methods have been previously validated (Domecq et al. 1995; Chapinal et al., 2009; Gibbons et al., 2014) and adapted from the Canadian Code of Practice for the Care and Handling of Dairy Cattle (National Farm Animal Care Council, 2009) and from Gibbons et al. (2012) and Vasseur et al. (2013). The training workshop described in this manuscript was used to train assessors for the National Dairy Study 2015 (Bauman et al., 2018). The workshop was designed to train 14 assessors over a 3 -d period for the 6 ABM: HI, LM in freestall and tiestall facilities, BCS, udder, flank, and leg CLN. Six weeks after the original training, inter-rater agreement for LM was assessed again due to lower inter-rater agreement at the end of the 3 -d workshop when compared with other ABM.
All scoring systems were modified before the start of the workshop so that the score or outcome was classified as acceptable or unacceptable, creating dichotomous outcomes for all ABM. Two-point systems have been shown to improve agreement among trainees (Knierim and Winckler, 2009) and are the method for the national proAction Animal Care Assessment Program in 2017 (Dairy Farmers of Canada, 2015). A simplified breakdown of the dichotomous scoring system for each ABM is presented in Table 1. The gait of cows housed in freestalls was evaluated on a 5-point lameness score (LS), where the acceptable threshold was less than 3 (Flower and Weary 2006). Cows that were housed in tiestalls were evaluated using in-stall lameness scoring (SLS; Leach et al., 2009). A video that describes the LS and SLS with example behaviors can be found online (http://www.nationaldairystudy.ca/videos). The SLS detection protocol has been shown to be a valid measurement for lameness when compared with gait scoring (Gibbons et al., 2014; Palacio et al., 2017).

\section{Training Workshop Participants}

All training was carried out by one of the authors (C. G. R. Nash), an experienced dairy welfare assessor who had received intensive welfare training and had professionally evaluated more than 200 herds at the time of the study using the same methods. This trainer was labeled as the expert in our study. The primary investigator (S. L. Croyle), who was individually trained by the expert before the workshop, also provided training assistance and workshop coordination. The 14 trainees were undergraduate university students $(\mathrm{n}=3)$, veterinary students $(\mathrm{n}=7)$, and veterinary technicians $(\mathrm{n}=$ 4) from the 5 Canadian Colleges of Veterinary Medicine and had a range of minimal or moderate to no previous training on evaluating these ABM. The purpose of the training was to standardize the welfare-scoring methodology to be used as one part of the comprehensive National Dairy Study (Bauman et al., 2018).

\section{Training Methodology}

A 3-d workshop was used to train and evaluate assessors for accuracy (ability to correctly identify an ABM) and inter-rater agreement. An additional training and practice video for LM was sent to the trainees $3 \mathrm{wk}$ after the workshop, and a follow-up assessment was performed 6 wk after the workshop. The training method was broken into sessions A through $\mathrm{L}$, described in Table 2, which highlights specific activities conducted during training period. Table 2 also provides the time allotted and the location required for each session, as well as the number of cows or the number of cow im- 
Table 1. Simplified descriptions ${ }^{1}$ of the dichotomous scores for acceptable and unacceptable animal based measures of welfare used to train assessors for the National Dairy Study

\begin{tabular}{|c|c|c|}
\hline $\begin{array}{l}\text { Animal-based } \\
\text { measure }\end{array}$ & Acceptable & Unacceptable \\
\hline Hock injuries & $\begin{array}{l}\text { No swelling with minor or no hair loss (score } 0 \text { ) } \\
\text { or } \\
\text { No swelling with bald area (score } 1 \text { ) }\end{array}$ & $\begin{array}{l}\text { Minor swelling or broken skin or scab (score } 2 \text { ) } \\
\text { or } \\
\text { Major swelling (thickness } \geq 2.5 \mathrm{~cm} \text { ), with or without bald } \\
\text { area or lesion (score } 3 \text { ) }\end{array}$ \\
\hline BCS & $\begin{array}{l}\text { Corrugation visible to more than half the length of the } \\
\text { short rib from the tip to the spine }\end{array}$ & $\begin{array}{l}\text { Corrugation visible to less than or equal to half the length } \\
\text { of the short rib from the tip to the spine }\end{array}$ \\
\hline Lameness (free) & $\begin{array}{l}\text { Score } \leq 2 \text { on a standard } 5 \text { point locomotion score, } \\
\text { indicating no limp }\end{array}$ & $\begin{array}{l}\text { Score } \geq 3 \text { on a standard } 5 \text { point locomotion score, } \\
\text { indicating a mild limp to severe limp }\end{array}$ \\
\hline Lameness $(\text { tie })^{2}$ & $\begin{array}{l}\text { Fewer than } 2 \text { out of } 4 \text { behavioral indicators for lameness } \\
\text { were observed (i.e., rest, edge, shift, uneven steps) }\end{array}$ & $\begin{array}{l}\text { Two or more out of the } 4 \text { behavioral indicators for } \\
\text { lameness were observed (i.e., rest, edge, shift, uneven steps) }\end{array}$ \\
\hline $\begin{array}{l}\text { Cleanliness: leg, } \\
\text { flank, or udder }\end{array}$ & Little or no manure to minor splashing & $\begin{array}{l}\text { Distinct plaques with hair showing through, to a solid } \\
\text { manure plaque }\end{array}$ \\
\hline
\end{tabular}

${ }^{1}$ Detailed photographic descriptions of animal-based measures of welfare listed can be viewed in the Animal Comfort Tool at www.dairyresearch .ca (Dairy Research Portal, 2018).

${ }^{2}$ The stall lameness score indicators of lameness are described as (1) edge: cow places hoof or hooves on edge of stall); (2) rest: cow lifts a hoof or hoof heel off of the ground and replaces it to the same location; (3) shift: (regular, repeated shifting of weight from one hoof to another); and (4) uneven steps: more rapid movement by one foot compared with the other or by an evident reluctance to bear weight on a particular foot when the cows was moved side to side (Leach et al., 2009; Dairy Research Portal, 2018).

ages or videos used. The sample size for cows tested was based on availability of resources, time, and confidence during training, and arranged to ensure that trainees were understanding the concepts. For the sessions that were analyzed for agreement, a minimum sample size of 20 was used as recommended in the Handbook of Interrater Reliability (Gwet, 2014). Specific details from sessions listed in Table 2 are described below, to both clarify methods and describe the parts of the training that were emphasized during the workshop.

Day 1. Sessions A through D included background education and scoring protocol overviews for each ABM, lasting approximately $3 \mathrm{~h}$. During this session, instruction for each ABM followed the same template: (1) protocol overview with written and graphic description of each score for the ABM, (2) diagrams and photos to explain where the assessor should stand depending on farm layout, (3) overview of the data entry process (spreadsheets on tablet computers), and (4) interactive learning. The interactive learning was conducted through group quizzes using projected images and video, where the 14 trainees were asked to vote by a show of hands if they would score a cow as acceptable or unacceptable for each ABM. These show-of-hands sessions were only 1 part of the training and occurred before individual testing to promote discussion and highlight discrepancies between trainees. In this way, trainees could practice with immediate feedback from the trainers. During the group quizzes, images or video were displayed, incorporating a range of all possible scores. The distribution of cow scores shown during image and video projection was $50 \%$ unacceptable and $50 \%$ acceptable for each ABM category. Following the group quiz, dialog took place between the trainees and the expert to discuss, and ultimately resolve, any discrepancies in scores given.

During sessions A, B, C, and D training emphasis was placed on the acceptable or unacceptable cutoffs for each ABM. For hocks, the focus was between a score of 1 and 2, giving attention to broken skin or scabs, where the expert described in detail that a very small scab received the same unacceptable score as a much larger open lesion. In addition, the expert emphasized investigating the hocks by touch, because a piece of manure in a photograph or during live scoring can look similar to a scab. During session B, which focused on BCS, emphasis was placed on recognizing the cutoff for 2 or $>2$, using images to highlight the corrugations along the short ribs. In session C, which addressed cleanliness scoring for flank, legs, and udders, emphasis was placed on distinguishing a splash from manure plaques. The majority of d 1 was spent on session D, focused on LS and SLS. Emphasis was placed on distinguishing a cow with a LS score of 2 (no limp) from a cow with LS of 3 (slight limp).

Day 2. Sessions E, F, G, and H (Table 2) took place at the same dairy farm. Session $F$ had each trainee individually assess cows $(\mathrm{n}=20)$ in tiestalls for all ABM except lameness. To begin, all trainees simultaneously stood behind a different cow and then moved to the 
next until all cows were scored. These 20 cows were not the same cows used in practice session E; thus, the trainees were naïve to this cow population for testing purposes. Lameness was not assessed due to the perceived distress and inaccuracy of re-evaluating each cow 14 times in succession using the SLS technique. This is one of the practical limitations to live scoring cows for in-stall lameness during training. The scores from session $\mathrm{F}$ generated the data for the d-2 agreement scores in the hock evaluations (Table 3). Sessions $\mathrm{G}$ and $\mathrm{H}$ consisted of expert-driven LS and SLS review and practice. Cows were observed in session H (LS; $\mathrm{n}=10$, SLS; $\mathrm{n}=5$ ) by the entire trainee group at one time. The expert and the principal investigator moved a cow, and students were asked to indicate if they scored the cows as acceptable or unacceptable.
The expert explained the rationale for the score given and discrepancies were discussed.

Day 3. During session I, trainees individually evaluated all ABM, except LM, on cows $(\mathrm{n}=33)$ in a freestall barn using headlocks using the same rotation method as session $\mathrm{F}$. This session generated data for the d-3 agreement scores for the hock evaluations (Table 3). Session J took place in a classroom, where trainees performed LS $(\mathrm{n}=20)$ and SLS $(\mathrm{n}=7)$ for videos of 27 cows. Results from this session were used to generate Lameness Evaluation 1 scores (Table 4).

At the conclusion of the 3-d workshop, an initial assessment of $\kappa$ scores gauged inter-rater agreement to determine if further training was required. A followup training and practice video (session $\mathrm{K}$ ) was sent to trainees $3 \mathrm{wk}$ after the workshop, before trainees

Table 2. Description of methods for a workshop and follow-up activities to train assessors in animal-based measures of welfare in dairy cows

\begin{tabular}{|c|c|c|c|c|c|c|}
\hline Time & Session & Location & Source & Activity & $\begin{array}{l}\text { Time } \\
(\min )\end{array}$ & $\begin{array}{l}\text { Cows } \\
\text { scored }\end{array}$ \\
\hline \multirow[t]{4}{*}{ Day 1} & $\mathrm{~A}$ & Classroom & Images & $\begin{array}{l}\mathrm{HI}^{1} \text { : methods review }+ \text { group quiz with } \mathrm{SH}^{2}+\mathrm{Q} \& \mathrm{~A}^{3} \\
\text { session. }\end{array}$ & 45 & 20 \\
\hline & B & Classroom & Images & $\begin{array}{l}\text { BCS: methods review + group quiz with SH + Q\&A } \\
\text { session. }\end{array}$ & 15 & 10 \\
\hline & $\mathrm{C}$ & Classroom & Images & $\begin{array}{l}\mathrm{CLN}^{4} \text { (flank, udder, leg): methods review + group quiz } \\
\text { with } \mathrm{SH}+\mathrm{Q} \& \mathrm{~A} \text { session. }\end{array}$ & 20 & 10 \\
\hline & $\mathrm{D}$ & Classroom & Videos & $\begin{array}{l}\mathrm{LM}^{5} \text { (LS, SLS): methods review + group quiz with } \mathrm{SH}+ \\
\text { Q\&A session. }\end{array}$ & 105 & 15 \\
\hline \multirow[t]{4}{*}{ Day 2} & $\mathrm{E}$ & Farm X & Locked cows & $\begin{array}{l}\text { In groups of } 3 \text { or } 4 \text {, trainees assessed hock, BCS, and } \\
\text { cleanliness orally. Cows were in headlocks or ties. Expert } \\
\text { walked around to answer questions as needed. }\end{array}$ & 60 & 30 \\
\hline & $\mathrm{F}$ & Farm X & Locked cows & $\begin{array}{l}\text { Individual trainees provided written scores for previously } \\
\text { unscored cows for hock, BCS, and cleanliness. Afterward, } \\
\text { expert discussed rationale for each score. }\end{array}$ & 30 & 20 \\
\hline & $\mathrm{G}$ & Farm X & Loose cows & $\begin{array}{l}\text { Expert-driven lameness review where expert discussed } \\
\text { rationale during scoring. }\end{array}$ & 20 & 10 \\
\hline & $\mathrm{H}$ & Farm X & $\begin{array}{l}\text { Loose/locked } \\
\text { cows }\end{array}$ & $\begin{array}{l}\text { Trainees performed evaluations of lameness (SLS + LS) } \\
\text { using SH. Immediately after a cow was scored, Q\&A } \\
\text { session followed [loose }(\mathrm{n}=10) \text { and locked }(\mathrm{n}=5)]\end{array}$ & 30 & 15 \\
\hline \multirow[t]{2}{*}{ Day 3} & I & Farm Z & Locked cows & $\begin{array}{l}\text { Individual trainees provided written scores for locked/ } \\
\text { tied cows for hock, BCS, and clean. Afterward, expert } \\
\text { discussed rationale for each cow score. }\end{array}$ & 45 & 33 \\
\hline & $\mathrm{J}$ & Classroom & Videos & $\begin{array}{l}\text { Individual trainees performed written evaluations on cows } \\
\text { for lameness (SLS + LS). Afterward, trainees compared } \\
\text { their scores to the expert. A Q\&A session followed. }\end{array}$ & 45 & 27 \\
\hline $\begin{array}{l}3 \text { wk } \\
\text { later }\end{array}$ & $\mathrm{K}$ & Online & Videos & $\begin{array}{l}\text { Expert-driven lameness review where expert discussed } \\
\text { rationale during the scoring of } 16 \text { cows. An additional } 9 \\
\text { practice cows could be scored by trainees, with the correct } \\
\text { answer provided immediately after each cow. }\end{array}$ & 20 & 25 \\
\hline $\begin{array}{l}6 \text { wk } \\
\text { later }\end{array}$ & $\mathrm{L}$ & Online & Videos & $\begin{array}{l}\text { Individual trainees performed written evaluations on cows } \\
\text { for gait. }\end{array}$ & 35 & 37 \\
\hline
\end{tabular}

${ }^{1}$ Hock indicates hock injury scoring.

${ }^{2} \mathrm{SH}$ indicates a show of hands method: an activity where assessor raised their hands to classify cows as acceptable or unacceptable.

${ }^{3} \mathrm{Q} \& \mathrm{~A}$ refers to question and answer sessions.

${ }^{4} \mathrm{CLN}$ indicates hygiene scoring for flank, udder, and leg.

${ }^{5}$ LM was lameness scoring for both locomotion scoring (LS) and tiestall lameness scoring (SLS). 
Table 3. Trainee evaluations for hock injury score agreement between an expert and 14 trainees on d 2 of training compared with d 3 of training, based on categorizing hock scores as acceptable or unacceptable

\begin{tabular}{|c|c|c|c|c|c|c|}
\hline \multirow[b]{2}{*}{ Expert $\times$ Trainee } & \multicolumn{2}{|c|}{$\begin{array}{l}\text { Hock evaluation } 1 \\
(20 \text { cows scored })\end{array}$} & \multicolumn{4}{|c|}{$\begin{array}{c}\text { Hock evaluation } 2 \\
\text { (33 cows scored })\end{array}$} \\
\hline & $\begin{array}{c}\text { d } 2 \\
\text { Agreement }^{1}(\%)\end{array}$ & $\begin{array}{c}\text { d } 2 \\
\text { Byrt's } \kappa^{2}\end{array}$ & $\begin{array}{c}\text { d } 3 \\
\text { Agreement (\%) }\end{array}$ & $\begin{array}{c}\text { d } 3 \\
\text { Byrt's } \kappa^{2}\end{array}$ & $\begin{array}{c}\text { d } 3 \\
\text { Cohen } \kappa^{3}\end{array}$ & $\mathrm{SE}^{4}$ \\
\hline $\mathrm{E} * \mathrm{~T} 1$ & 95 & 0.90 & 91 & 0.82 & 0.68 & 0.17 \\
\hline $\mathrm{E} * \mathrm{~T} 2$ & 50 & 0.00 & 82 & 0.64 & 0.68 & 0.17 \\
\hline $\mathrm{E} * \mathrm{~T} 3$ & 70 & 0.40 & 97 & 0.94 & 0.87 & 0.13 \\
\hline $\mathrm{E} * \mathrm{~T} 6$ & 75 & 0.50 & 88 & 0.76 & 0.60 & 0.17 \\
\hline $\mathrm{E} * \mathrm{~T} 7$ & 80 & 0.60 & 97 & 0.94 & 0.87 & 0.13 \\
\hline $\mathrm{E} * \mathrm{~T} 8$ & 80 & 0.60 & 97 & 1.00 & 1.00 & 0.00 \\
\hline $\mathrm{E} * \mathrm{~T} 9$ & 80 & 0.60 & 85 & 0.70 & 0.46 & 0.20 \\
\hline $\mathrm{E} * \mathrm{~T} 10$ & 95 & 0.90 & 97 & 0.94 & 0.87 & 0.13 \\
\hline $\mathrm{E} * \mathrm{~T} 11$ & 90 & 0.80 & 91 & 0.82 & 0.68 & 0.17 \\
\hline $\mathrm{E} * \mathrm{~T} 12$ & 90 & 0.80 & 97 & 0.94 & 0.87 & 0.13 \\
\hline
\end{tabular}

${ }^{1}$ Agreement indicates crude percent agreement of classification between the trainee and expert.

${ }^{2}$ Byrt's $\kappa=$ prevalence-adjusted score of agreement of classification between the trainee and the expert.

${ }^{3}$ Cohen's $\kappa=$ bias-adjusted score of agreement of classification between the trainee and the expert.

${ }^{4} \mathrm{SE}$ for the Cohen's $\kappa$ for each trainee on their final assessment.

began work in the field. The video reviewed both LS and SLS techniques $(\mathrm{n}=16)$ and provided $(\mathrm{n}=9)$ practice cows. Six weeks after the workshop, and $3 \mathrm{wk}$ into field collection, all trainees actively involved in lameness evaluation during the National Dairy study $(\mathrm{n}=13)$ took part in session L. Session L consisted of another video assessment, where trainees assessed cows
( $\mathrm{n}=37$ ) for LM. This session occurred 4 wk into the National Dairy Study data collection (6 wk after initial training), and was used to ensure that assessors were accurately identifying $\mathrm{ABM}$ and that high inter-rater agreement was maintained. The scores given by the trainees to the cows in the video session generated the data for Lameness Evaluation 2 (Table 4).

Table 4. Trainee evaluations for lameness scoring agreement between an expert and 14 trainees on d 3 of training (lameness evaluation 1 ) and 6 wk later (lameness evaluation 2)

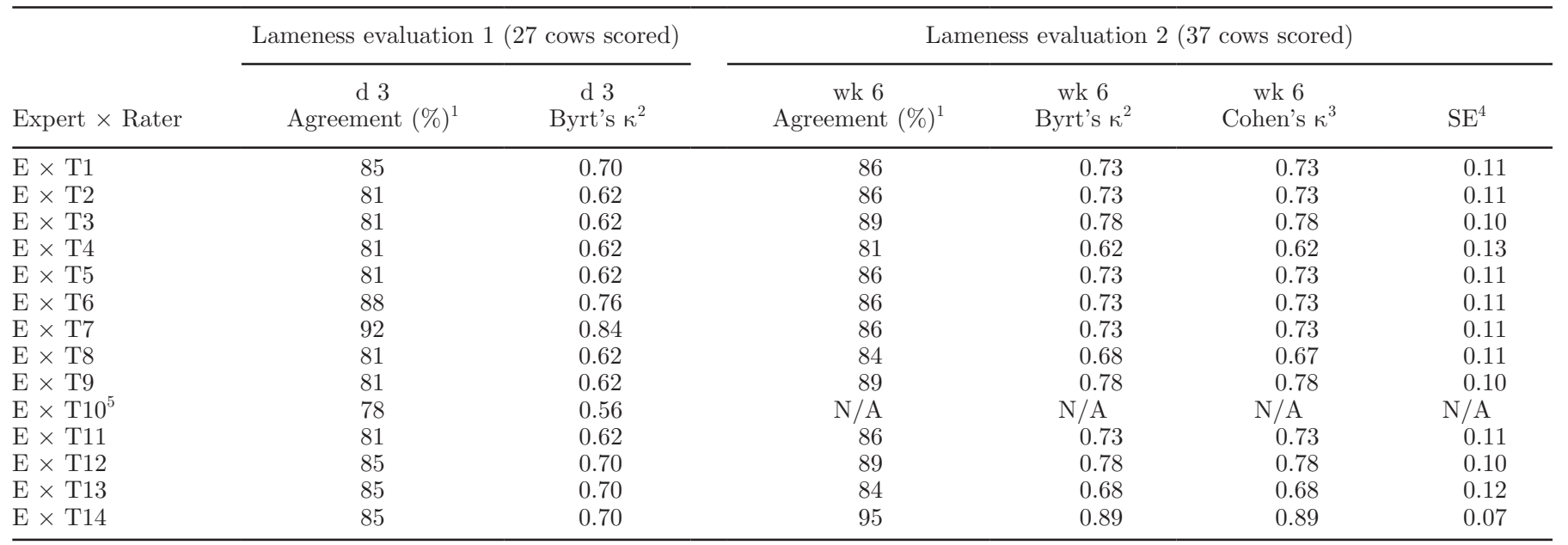

${ }^{1}$ Agreement indicates crude percent agreement of classification between the trainee and expert.

${ }^{2}$ Byrt's $\kappa=$ the prevalence-adjusted score of agreement of classification between the trainee and the expert.

${ }^{3}$ Cohen's $\kappa=$ bias-adjusted score of agreement of classification between the trainee and the expert.

${ }^{4} \mathrm{SE}$ for Cohen's $\kappa$ for each trainee on their final assessment.

${ }^{5}$ Trainee 10 did not evaluate cows for lameness in the field following initial training, so follow-up was not assessed. 
Table 5. Agreement of classification at the individual (expert-trainee) and at the group level (trainee-trainee) of animal-based measures among 14 trainees during and after a 3 -d workshop

\begin{tabular}{|c|c|c|c|c|c|c|c|}
\hline $\begin{array}{l}\text { Animal-based } \\
\text { measure }\end{array}$ & Time $^{1}$ & $\begin{array}{l}\text { No. of } \\
\text { trainees }\end{array}$ & $\begin{array}{c}\text { Total } \\
\text { scores }^{2}\end{array}$ & $\begin{array}{l}\text { Proportion } \\
\text { scored } \\
\text { acceptable } \\
\quad(\%)^{3}\end{array}$ & $\begin{array}{l}\text { Crude agreement }^{5} \\
\text { mean }[\text { minimum } \\
\quad-\text { maximum }]\end{array}$ & $\begin{array}{c}\text { Byrt's } \kappa^{4} \\
\text { mean }[\text { minimum } \\
- \text { maximum] }\end{array}$ & $\begin{array}{c}\text { Fleiss's } \kappa^{6} \\
\text { (group agreement) } \\
(\mathrm{CI})\end{array}$ \\
\hline \multirow[t]{2}{*}{ Hock injury } & $\mathrm{d} 2$ & 14 & 280 & 82 & $80[50$ to 95$]$ & $0.62[0.00$ to 0.91$]$ & $0.55(0.51$ to 0.58$)$ \\
\hline & d 3 & 14 & 462 & 74 & $91[82$ to 97$]$ & $0.85[0.64$ to 1.00$]$ & $0.66(0.62$ to 0.70$)$ \\
\hline \multirow[t]{2}{*}{ Lameness } & d 3 & 14 & 378 & 59 & $81[78$ to 92$]$ & $0.66[0.56$ to 0.84$]$ & $0.43(0.39$ to 0.47$)$ \\
\hline & wk 6 & 13 & 429 & 57 & $86[81$ to 95$]$ & $0.74[0.62$ to 0.89$]$ & $0.66(0.61$ to 0.69$)$ \\
\hline \multirow[t]{2}{*}{$\mathrm{BCS}$} & d 2 & 14 & 280 & 95 & $100[95$ to 100$]$ & $0.99[0.95$ to 1.00$]$ & $1.00(0.96-1.00)$ \\
\hline & d 3 & 14 & 462 & 96 & $100[100$ to 100$]$ & $1.00[1.00$ to 1.00$]$ & $1.00(0.97-1.00)$ \\
\hline \multirow[t]{2}{*}{ Leg cleanliness } & d 2 & 14 & 280 & 75 & $100[100$ to 100$]$ & $1.00[1.00$ to 1.00$]$ & $1.00(0.96-1.00)$ \\
\hline & d 3 & 14 & 462 & 91 & $97[91$ to 100$]$ & $0.96[0.87$ to 1.00$]$ & $0.88(0.84-0.91)$ \\
\hline \multirow[t]{2}{*}{ Flank cleanliness } & $\mathrm{d} 2$ & 14 & 280 & 85 & $100[100$ to 100$]$ & $1.00[1.00$ to 1.00$]$ & $1.00(0.96-1.00)$ \\
\hline & d 3 & 14 & 462 & 94 & $96[91$ to 100$]$ & $0.96[0.87$ to 1.00$]$ & $0.89(0.85-0.92)$ \\
\hline \multirow[t]{2}{*}{ Udder cleanliness } & $\mathrm{d} 2$ & 14 & 280 & 90 & $100[100$ to 100$]$ & $1.00[0.95$ to 1.00$]$ & $1.00(0.96-1.00)$ \\
\hline & d 3 & 14 & 462 & 87 & $97[91$ to 97$]$ & $0.90[0.82$ to 0.94$]$ & $0.90(0.86-0.93)$ \\
\hline
\end{tabular}

${ }^{1}$ Time indicates the day of training within or following the 3 -d workshop.

${ }^{2}$ Total number of cows scored $\times$ No. Trainees who participated in that portion of the workshop.

${ }^{3}$ Proportiong scored acceptable is the percent of total cows that were identified as acceptable by the expert.

${ }^{4}$ Byrt's $\kappa$ indicates expert-rater agreement.

${ }^{5}$ Crude agreement indicates the unadjusted \% agreement of classification.

${ }^{6}$ Fleiss's $\kappa$ indicates agreement among all of the assessors.

\section{Statistical Methods}

The training assessment results were evaluated for agreement using tests that measure accuracy and interrater reliability of the trainees. Kappa is a statistical measure that assesses the inter-rater reliability between 2 different raters (Cohen, 1968; Byrt et al., 1993) or among a group of raters (Fleiss, 1971) when they are assigning categorical ratings to specific outcomes. The $\kappa$ systems use an agreement scale of poor $(<0)$, slight $(0.01-0.20)$, fair $(0.21-0.40)$, moderate $(0.41-0.60)$, substantial $(0.61-0.80)$, or almost perfect $(0.81-1.00)$ (Landis and Koch, 1977). The effectiveness of the training was evaluated after each assessment by calculating Byrt's $\kappa$ between each trainee and the expert to look at trainee-expert agreement. Byrt's $\kappa$ was the most appropriate inter-rater agreement test for the data set due to cow score distributions during on-farm scoring. However, Cohen's $\kappa$ was also reported for the final inter-rater agreement session as a best practice for reporting, and as useful comparison to other studies where Cohen's $\kappa$ is used. The effectiveness of the training was also assessed by Fleiss's $\kappa$ to determine group-level inter-rater agreement among the assessor group (more than 2 raters). In addition, a $t$-test was used to examine the mean difference in the Byrt's $\kappa$ between $\mathrm{d} 2$ and 3 for $\mathrm{HI}$ and between $\mathrm{d} 3$ and wk 6 for LM. This test was done to demonstrate whether the trainees had significantly improved between the 2 testing sessions. The $\kappa$ scores for BCS and CLN of the leg, flank, and udder were already very high on both d
2 and 3 (Table 5); therefore, detailed individual experttrainee improvement between days was not reported. For both the first and second analyzed hock evaluations (Table 3), the trainee and expert were compared using multiple methods. Crude percent agreement was calculated, which is simply the sum of all responses where both the expert and trainee agreed in scoring an ABM as acceptable or unacceptable. In addition, the trainee and expert were compared using Byrt's and Cohen's $\kappa$, which both account for agreement by chance. The $\kappa$ analyses were calculated using SAS version 9.2 (SAS Institute Inc., Cary, NC). A specific macro was used to calculate Fleiss $\kappa$ (Chen et al., 2005).

\section{RESULTS AND DISCUSSION}

By using the described methods, very good agreement was achieved both at the group level and between the trainees and expert for all ABM. Traditionally, for studies exploring ABM, a $\kappa$ score of $>0.60$ is the lowest cutoff used to report a high level of agreement (Gibbons et al., 2012; Vasseur et al. 2013). Byrt's $\kappa$ uses an index of agreement between the trainees and the expert, and adjusts kappa for differences in prevalence of the conditions (i.e., the number of acceptable vs. unacceptable cows in the population; Byrt et al., 1993). Cohen's $\kappa$ attempts to give an agreement score with an adjustment for the possibility that raters might guess on some cows due to uncertainty, but estimates are unrepresentatively low when the prevalence of an outcome is low (Hallgren, 2012). Therefore, it is suggested that 
both Cohen's $\kappa$ and Byrt's $\kappa$ scores are reported when comparing raters for agreement (Hallgren, 2012). Byrt's $\kappa$ was used because trainees were evaluating cows on 2 farms for live training purposes, and the distribution of cow scores could not be predetermined. For some $\mathrm{ABM}$ in the study (BCS and CLN), the prevalence of unacceptable scores was very low. Byrt's $\kappa$ helps to address the limitation of live training where there are no guarantees of an even distribution of cows in each score category for each ABM. Fleiss's $\kappa$ was also calculated to evaluate the overall group inter-rater agreement for both HI and LM, to demonstrate that the group of 14 trainees was repeatable (precise) when compared against each other. Evaluating group-level agreement is important when different individuals are collecting and comparing data in different regions or on different farms, which was the method to be used during the 2015 Canadian National Dairy Study (Bauman et al., 2018).

Byrt's $\kappa$ scores (Table 5) demonstrate that, on both d 2 and 3 of training, all 14 trainees achieved almost perfect agreement $(>0.95)$ for acceptable versus unacceptable BCS and leg, udder, and flank CLN. These results indicate that, although less time was spent on the training process for discriminating unacceptable from acceptable cows for BCS and CLN compared with other ABM, the time allotted was adequate for achieving almost perfect agreement.

During session $\mathrm{F}$, the trainees were able to achieve an HI average Byrt's $\kappa$ of 0.62 (substantial agreement), ranging from 0.0 (less than chance agreement) to 0.90 (almost perfect agreement; Table 5). By session I of training, trainees achieved an HI Byrt's $\kappa$ of 0.83 (almost perfect agreement), ranging from 0.64 (substantial agreement) to 0.94 (almost perfect agreement; Table 5). The Byrt's $\kappa$ scores improved by $0.21(P<$ $0.005)$ from session $\mathrm{F}$ to session I for HI, indicating that after both a classroom and live training session, a minimum of 1 additional live scoring session should take place for training in the evaluation of hock scores. During session $J$, the trainees were able to achieve an average LM Byrt's $\kappa$ of 0.66 (substantial agreement) for lameness evaluation, ranging from 0.56 (moderate agreement) to 0.84 (almost perfect agreement; Table 5). The trainee $(\mathrm{n}=1)$ that fell into the moderate category chose not to score any cows for lameness during the national study, and was excluded from session L ( $\mathrm{n}=$ 13). In session $\mathrm{L}$, trainees $(\mathrm{n}=13)$ achieved a mean $\mathrm{LM}$ Byrt's $\kappa$ of 0.74 (substantial agreement), ranging from 0.62 (substantial agreement) to 0.89 (almost perfect agreement; Table 5). The mean Byrt's $\kappa$ scores for LM increased by $0.06(P<0.005)$ from $\mathrm{d} 3$ of the workshop for lameness to 6 wk later, suggesting that trainees were improving their scoring techniques through practice and that the postworkshop refresher video $3 \mathrm{wk}$ into the study helped solidify lameness-evaluation techniques. The change in inter-rater agreement between each assessor and the expert for both hock injury and lameness scores are depicted in Figure 1 and Figure 2, respectively. The second assessment resulted in the vast

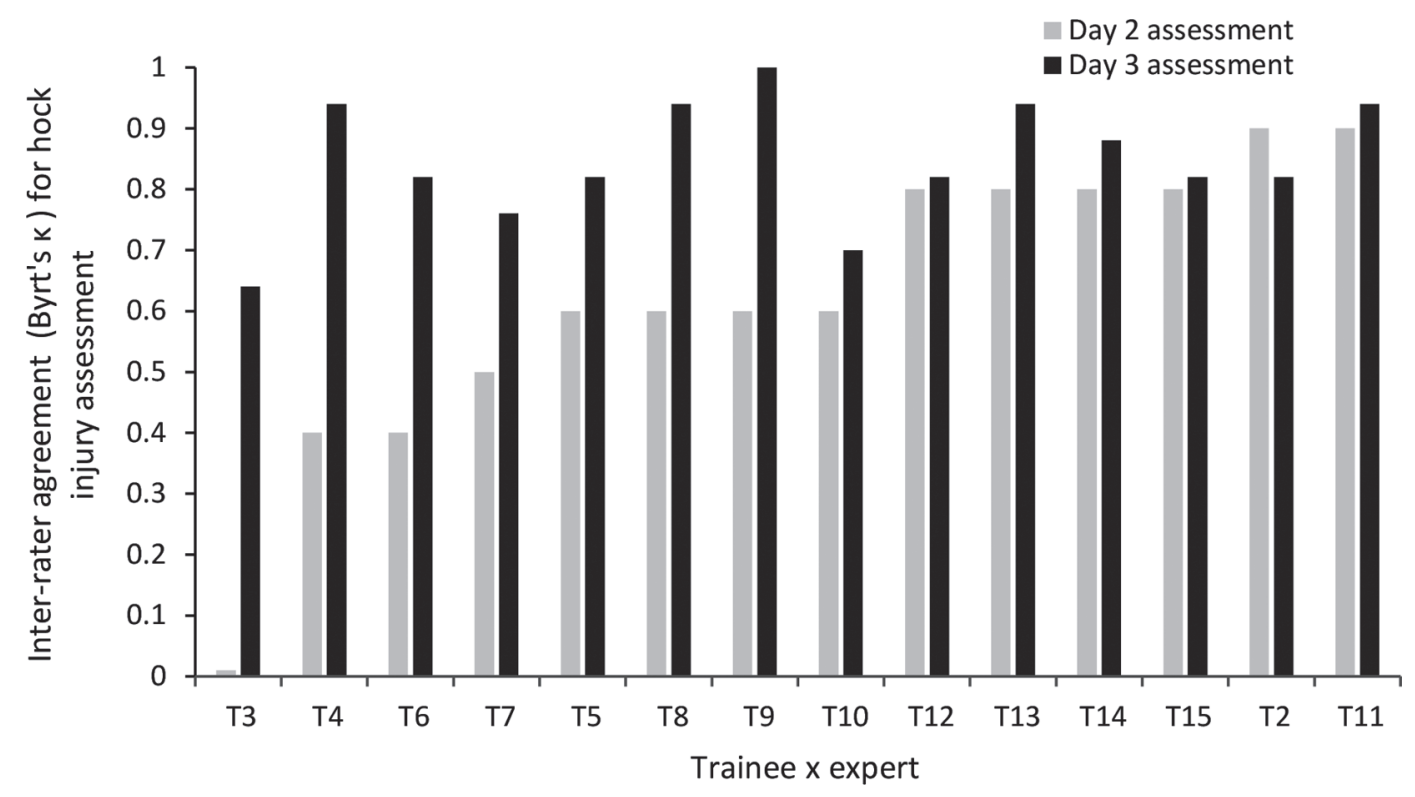

Figure 1. Change in inter-rater agreement score (Byrt's $\kappa$ ) for hock injuries between the expert and each trainee. The gray line demonstrates the trainee's first assessment after training (d 2), and the black line represents the trainee's second assessment (d 3), which occurred after reviewing results of the first assessment. Trainees and expert recorded hock injuries as acceptable or nonacceptable. 
majority of trainees improving, especially when initial scores were low.

Overall agreement among all 14 trainees was assessed for $\mathrm{HI}$ and LM and is represented using Fleiss's $\kappa$ measures (Table 5). The Fleiss's $\kappa$ agreement level for HI among the 14 trainees was 0.55 on $\mathrm{d} 2$ and 0.66 on $\mathrm{d}$ 3 of the workshop indicating that, similar to Byrt's $\kappa$ scores, a second day of live scoring is recommended for achieving substantial agreement. Fleiss's $\kappa$ agreement level for LM among the 14 assessors was 0.43 at the end of the 3-d workshop and 0.66 after the follow-up training and practice video, indicating that 1 classroom session and 1 live session should be followed up with additional training and practice to ensure higher levels of agreement among the raters.

Trainees were able to achieve both substantial accuracy and high group-level inter-rater reliability, as shown through Byrt's and Fleiss's $\kappa$ measures. The discussions that took place during the classroom group quizzes, as well as following the hock injury evaluation on d 2 and 3 and lameness evaluation on d 3, allowed trainees to sharpen their skills by learning from their mistakes. This was demonstrated by continuous improvement throughout the training period (Table 5). Previous researchers have recommended evaluating agreement among trainees on d 1, 2, 6, 7, and, if necessary, 8 of a training period, as well as 4 wk and 15 wk after training to ensure consistency and accuracy over time (Gibbons et al., 2012; Vasseur et al., 2013). The cost and feasibility of evaluating trainees that frequently may not be practical for all research purposes.
One strength of the current workshop design was that it demonstrated that in a 3-d workshop, a minimum of almost perfect agreement (Byrt's $\kappa$ of 0.81-0.99) could be achieved for each ABM except LM. Substantial agreement (0.61-0.80), the cutoff most commonly used as an acceptable inter-rater agreement, was reached for LM (Thomsen et al., 2008; Gibbons et al., 2012). Although LM had relatively lower $\kappa$ scores compared with the other measures, agreement in LM appeared to improve over time with follow-up practice videos, indicating that future workshops should incorporate even more lameness videos in the initial training and assessment.

Although categories of classification were not distributed evenly in the current study, it was important that at least 1 cow was considered unacceptable for each ABM during each live training session to calculate Byrt's $\kappa$ scores. The HI and LM required the most time for the question and answer sessions, and therefore live scoring should be done that includes substantial variation in hock injuries and lameness so that a variety of scenarios can been seen and discussed. Using pictures and videos in addition to live cows is a way to increase variation in scores, as done in other training methods (Gibbons et al., 2012; Vasseur et al., 2013). For our study, pictures were only used in sessions A through E, for classroom training and classroom group-level quizzes. Trainees found the pictures and videos valuable and expressed the desire for more practice videos for lameness evaluation, which resulted in the wk 3 followup lameness practice video.

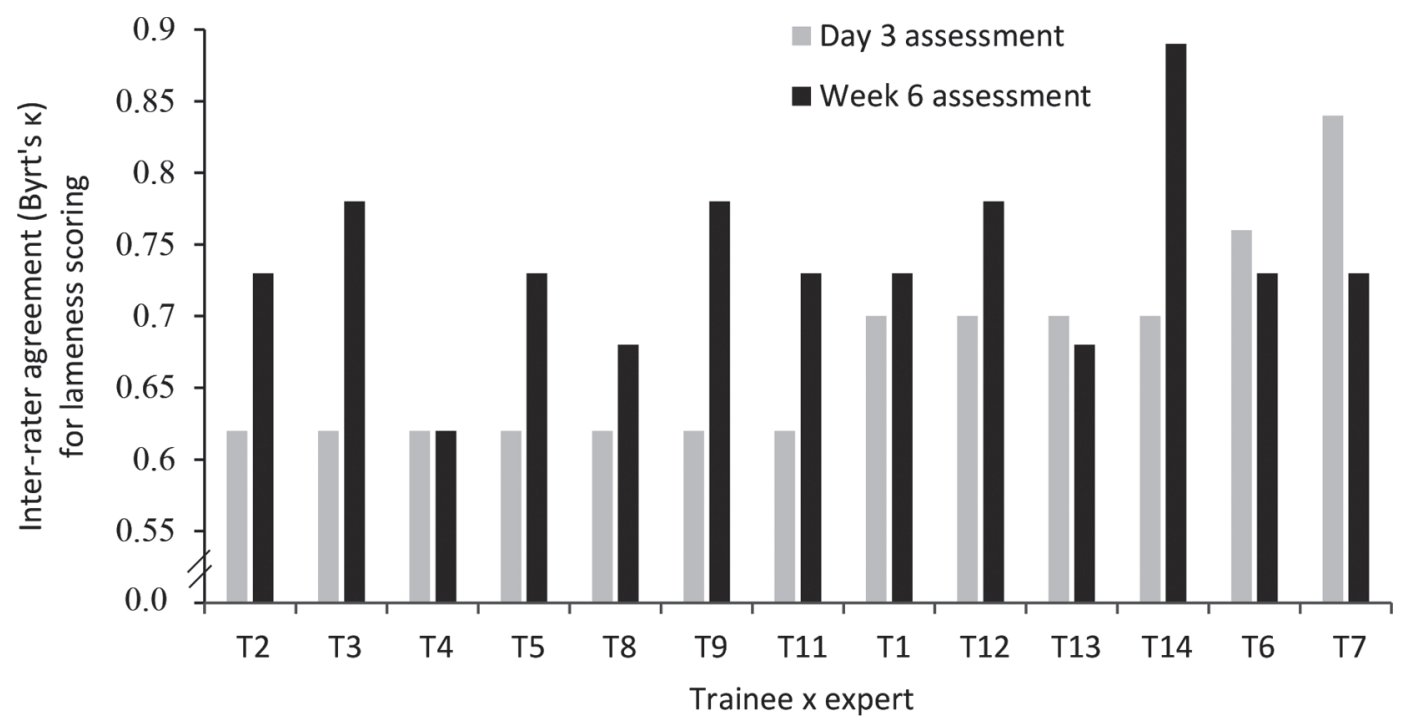

Figure 2. Change in inter-rater agreement score (Byrt's $\kappa$ ) for lameness between the expert and each trainee. The gray line demonstrates the trainee's first assessment after training (d 3), and the black line represents the trainee's second assessment (wk 6), which occurred after reviewing results of the first assessment, and viewing an additional training and practice video. Trainees and expert recorded lameness as acceptable or nonacceptable. 


\section{CONCLUSIONS}

The results of our study demonstrate that a training workshop comprised of multiple media (photos, video, and live training) can achieve acceptable grouplevel agreement for several ABM of welfare. This model resulted in crude agreement of at least $81 \%$ for each $\mathrm{ABM}$, and $\kappa$ scores $\geq 0.61$, which is the most commonly used $\kappa$ cutoff for acceptable inter-rater agreement in studies using multiple observers for subjective scoring methods. We recommend that training take place with intermittent testing so that trainees can learn from their mistakes as they progress through the training process. This type of workshop may be useful for training accurate observers with substantial inter-rater agreement for research, veterinary services, or for individuals involved in welfare assessments in dairy herds.

\section{ACKNOWLEDGMENTS}

Funding for this project was provided through the Dairy Cluster II by Dairy Farmers of Canada (Ottawa, Canada), Agriculture and Agri-Food Canada (Guelph, Canada), The Canadian Dairy Network (Guelph, Canada), and The Canadian Dairy Commission (Ottawa, Canada). Data analysis and preparation of the manuscript were done independently of the project funders. The authors declare no conflicts of interest. We thank the dairy farmers that offered their farms and time for our training workshop.

\section{REFERENCES}

Bauman, C. A., H. W. Barkema, J. Dubuc, G. P. Keefe, and D. F. Kelton. 2018. Canadian Dairy Study: Herd-level milk quality. J. Dairy Sci. 101:2679-2691.

Byrt, T., J. Bishop, and J. B. Carlin. 1993. Bias, prevalence and Kappa. J. Clin. Epidemiol. 46:423-429.

Canadian Dairy Research Portal. 2018. Animal Comfort Tool-Animal Outcome Measurement Protocols Injury, Cleanliness, Body Condition, and Lameness. Accessed Feb. 26, 2018. https://www .dairyresearch.ca/animal-comfort-tool.php.

Chapinal, N., A. M. De Passille, D. M. Weary, M. A. G. Von Keyserlingk, and J. Rushen. 2009. Using gait score, walking speed, and lying behavior to detect hoof lesions in dairy cows. J. Dairy Sci. 92:4365-4374.

Chen, B., D. Zaebst, and L. Seel. 2005. A macro to calculate kappa statistics for categorizations by multiple raters. SUGI 30 proceedings, April 10-13, 2005. Philadelphia, PA.

Cohen, J. 1968. Weighted kappa: Nominal scale agreement with provision for scaled disagreement or partial credit. Psychol. Bull. $70: 213-220$.
Dairy Farmers of Canada. 2015. proAction: Leading the way for sustainable dairy farming; providing assurance to customers about farm practices. Dairy Farmers of Canada, Ottawa, Ontario, Canada. Accessed Apr. 18, 2017. https://www.dairyfarmers.ca/ proaction\#animal-care.

Domecq, J. J., A. L. Skidmore, J. W. Lloyd, and J. B. Kaneene. 1995. Validation of body condition scores with ultrasound measurements of subcutaneous fat of dairy cows. J. Dairy Sci. 78:2308-2313.

Fleiss, J. L. 1971. Measuring nominal scale agreement among many raters. Psychol. Bull. 76:378-382.

Flower, F. C., and D. M. Weary. 2006. Effect of hoof pathologies on subjective assessments of dairy gait. J. Dairy Sci. 89:139-146.

Gibbons, J., D. B. Haley, J. H. Cutler, C. G. Nash, J. C. Zaffino Heyerhoff, D. Pellerin, S. Adam, A. Fournier, A. M. de Passillé, J. Rushen, and E. Vasseur. 2014. Technical note: A comparison of 2 methods of assessing lameness prevalence in tiestall herds. J. Dairy Sci. 97:350-353.

Gibbons, J., E. Vasseur, J. Rushen, and A. M. de Passillé. 2012. A training programme to ensure high repeatability of injury scoring of dairy cows. Anim. Welf. 21:379-388.

Gwet, K. L. 2014. Handbook of Inter-Rater Reliability: The Definitive Guide to Measuring the Extent of Agreement Among Raters. Advanced Analytics LLC, Gaithersburg, MD.

Hallgren, K. A. 2012. Computing inter-rater reliability for observational data: An overview and tutorial. Tutor. Quant. Methods Psychol. 8:23.

Knierim, U., and C. Winckler. 2009. On-farm welfare assessment in cattle: validity, reliability and feasibility issues and future perspectives with special regard to the Welfare Quality ${ }^{\circledR}$ approach. Anim. Welf. 18:451-458

Landis, J. R., and G. G. Koch. 1977. The measurement of observer agreement for categorical data. Biometrics 33:159-174.

Leach, K. A., S. Dippel, J. Huber, S. March, C. Winckler, and H. R. Whay. 2009. Assessing lameness in cows kept in tie-stalls. J. Dairy Sci. 92:1567-1574.

Lievens, F. 2001. Assessor training strategies and their effects on accuracy, interrater reliability, and discriminant validity. J. Appl. Psychol. 86:255-264.

National Farm Animal Care Council. 2009. Code of Practice for the Care and Handling of Dairy Cattle. Ottawa, Ontario, Canada. Accessed Jan. 18, 2015. http://www.nfacc.ca/pdfs/codes/dairy_code _of_practice.pdf.

Palacio, S., L. Peignier, C. Pachoud, C. Nash, S. Adam, R. Bergeron, D. Pellerin, A. M. de Passillé, J. Rushen, D. Haley, T. J. DeVries, and E. Vasseur. 2017. Technical note: Assessing lameness in tiestalls using live stall lameness scoring. J. Dairy Sci. 100:6577-6582.

Rushen, J., A. Butterworth, and J. C. Swanson. 2011. Animal behavior and well-being symposium: Farm animal welfare assurance: science and application. J. Anim. Sci. 89:1219-1228.

Thomsen, P. T., L. Munksgaard, and F. A. Tøgersen. 2008. Evaluation of a lameness scoring system for dairy cows. J. Dairy Sci. 91:119-126.

Vasseur, E., J. Gibbons, J. Rushen, and A. M. de Passillé. 2013. Development and implementation of a training program to ensure high repeatability of body condition scoring of dairy cows. J. Dairy Sci. 96:4725-4737.

Whay, H. R., D. C. J. Main, L. E. Green, and A. J. F. Webster. 2003. Animal-based measures for the assessment of welfare state of dairy cattle, pigs and laying hens: Consensus of expert opinion. Anim. Welf. 12:205-217. 